\title{
Validation and Benchmarking of a Wearable EEG Acquisition Platform for Real-World Applications
}

\author{
Olivier Valentin*, Mikaël Ducharme, Gabrielle Crétot-Richert, Hami Monsarrat-Chanon, \\ Guilhem Viallet, Aidin Delnavaz and Jérémie Voix.
}

\begin{abstract}
This paper presents the experimental validation of a readout circuit for the acquisition, amplification and transmission of extremely weak biopotentials with a focus on electroencephalography (EEG) signals. The device, dubbed CochlEEG, benefits from a low-power design for long-term power autonomy and provides configurable gain and sampling rates to suit the needs of various EEG applications. CochlEEG features high sampling rates, up to $4 \mathrm{kHz}$, low noise signal acquisitions, support for active electrodes and a potential for Wi-Fi data transmission. Moreover, it is lightweight, pocket-size and affordable, which makes CochlEEG suitable for wearable and real-world applications. The efficiency of CochlEEG in EEG data acquisition is also investigated in this paper. Auditory Steady State Responses (ASSR) acquisition results validate CochlEEG's capability in recording EEG with a signal quality comparable to commercial mobile or research EEG acquisition devices. Moreover, the results of an oddball paradigm experiment prove the capability of CochlEEG in recording Event-Related Potentials (ERP) and demonstrate its potential for Brain-Computer Interface (BCI) applications and electrophysiological research applications requiring higher temporal resolution.
\end{abstract}

Index Terms-Auditory steady-state response (ASSR), wearable EEG, event-related potentials (ERP).

\section{INTRODUCTION}

$\mathrm{R}^{\mathrm{s}}$ ECENT advances in the manufacturing of inconspicuous body sensors and miniaturized circuits as well as considerable developments in telecommunications and data analysis techniques have opened up new possibilities for the integration of sensors into various accessories worn in daily life, such as clothing, hats and shoes. Body sensors and wearables have various applications including health and safety monitoring, home rehabilitation, and early detection of disorders, to name a few examples [1].

Electroencephalography (EEG) sensors for measuring brain electrical activity are becoming a major category of bodyworn sensors with a variety of applications in clinical studies, medical diagnosis and neurological researches. EEG signals

O. Valentin*, M. Ducharme, G. Crétot-Richert, H. Monsarrat-Chanon, G. Viallet, A. Delnavaz and J. Voix are with the NSERC-EERS Industrial Research Chair in In-Ear Technologies, École de technonolgie supérieure, Montreal, QC, Canada. (*correspondence e-mail: m.olivier.valentin@gmail.com). can be measured by active or passive electrodes placed on or next to the scalp. These acquired signals must then be amplified and transmitted to a computer usually for further processing. Conventional EEG signal acquisition systems are bulky, which has impeded their integration into wearables and imposes serious limitations on EEG applications outside of a laboratory environment. Over the years, several mobile EEG signal acquisition systems have emerged. Some of these are commercialized as consumer products. For example, SMARTING ${ }^{\mathrm{TM}}$ (mBrainTrain, Belgrade, Serbia) is a small EEG device for real-time brain activity monitoring. ENOBIO ${ }^{\circledR}$ (Neuroelectrics, Barcelona, Spain) is a wearable wireless electrophysiological signal acquisition system for EEG recording. Also, the EEG-SMT board (Olimex Ltd, Plovdiv, Bulgaria) is available as an open-source software and hardware unit for recording EEG signals. Similarly, Cyton biosensing board (OpenBCI, Brooklyn, NY, USA) can be used to record brain, muscle and heart activity on a wireless opensource platform. Moreover, Cognionics Inc. (San Diego, CA, USA) offers a wide range of mobile EEG systems for both high-density and low-density EEG recordings. Finally, Brain Products (Munich, Germany) recently released LiveAmp, a high-quality mobile wireless EEG recording solution.

Aside from the commercialized products, some research groups have developed their own mobile EEG recording systems for more specific applications. For example, EEGu2 is an embedded device for brain/body signal acquisition developed and used in the context of human-in-the-loop cyber-physical systems [2]. BioDAQ (Biological Data Acquisition) is a low-cost system that has been developed by Rahman and Nasor for real time biomedical applications [3] and a wearable multi-channel EEG recording system featuring a compression algorithm is presented by Dufort et al. [4].

However, most mobile EEG products on the market share common downsides. They are expensive, ranging from several hundred dollars to more than tens of thousands, which may impose a serious financial barrier on neurological research studies. It can also prevent EEG systems from being widely used in Brain-Computer Interface (BCI) devices. Furthermore, oftentimes some commercial products do not provide the raw EEG data to the consumers, which makes them less interesting for research applications. In addition, the limited sampling rate (often $500 \mathrm{~Hz}$ and less) of commercial products prevents their 
use for specific electrophysiological research applications that require higher temporal resolution. Finally, many commercial EEG systems can only support a limited number of electrodes (under 5) and are not compatible with active EEG electrodes.

While these more recent portable EEG recording devices, developed by research groups and companies alike, answer innovative applications' need for less bulky equipment, they can be limiting compared to research grade equipment. Certain applications for wearable EEG devices might be impeded by these constraints. A more accessible and flexible system that could accommodate electrode technology while supporting multiple input channels as well as greater sampling frequencies and raw data output streams could be the solution for these applications.

This paper presents a wearable electronic device dubbed CochlEEG intended for the acquisition, amplification and transmission of EEG signals, designed in an attempt to overcome the previously described limitations. It provides eight channels that can connect to both passive and active EEG electrodes. Its battery-powered circuit board along with its lightweight and pocket-sized packaging, positions the CochlEEG as an ideal candidate for portable EEG. It is convenient to carry and would be easy to integrate into wearables. Moreover, it offers a very precise time resolution with sampling frequency reaching up to $4 \mathrm{kHz}$, which is required for applications such as Frequency Following Responses (FFR) among others. The objective of this research is to test and compare the EEG recording performance of CochlEEG. For that purpose, two EEG paradigms with high potential in the context of auditory research and BCI applications were chosen to evaluate the performance of CochlEEG to record EEG time series and frequency spectrums. In a first study, Auditory Steady-State Responses (ASSRs) recording performance with CochlEEG was tested and the spectral results were compared with those obtained by 1) Rotman MASTER research system (Baycrest Center, University of Toronto, Canada) as a conventional researchgrade EEG research system and 2) SMARTING ${ }^{\mathrm{TM}}$ as a wellknown, tested and proven, commercially available mobile EEG device. In a second study, Event-Related Potentials (ERP) from an auditory oddball paradigm were recorded using CochlEEG to demonstrate the capability of the proposed EEG acquisition platform for such applications in the temporal domain.

This paper is structured as follows: the equipment and experimental procedures are described in section II. ASSRs results and ERP results are presented in section III and discussed in section IV. Conclusions and the future works are given in section $\mathrm{V}$.

\section{METHODS}

Two studies were conducted and the methodologies employed are presented in this section. The first study is a comparison study between three EEG systems presented in table I, an ASSR paradigm was used. Previous work from Melnik et al. [5] developed a scientific approach to evaluate and compare EEG systems. To account for the systems
TABLE I

CHARACTERISTICS OF THE THREE DIFFERENT ACQUISITION SYSTEMS

\begin{tabular}{|c|c|c|c|c|c|}
\cline { 2 - 6 } \multicolumn{1}{c|}{} & $\begin{array}{c}\text { SAMPLING } \\
\text { FREQUENCY }\end{array}$ & RESOLUTION & $\begin{array}{c}\text { InPUT NOISE } \\
\text { (RMS) }\end{array}$ & WEIGHT & Size \\
\hline SMARTING & 250 to $500 \mathrm{~Hz}$ & 24 bits & $353 \mathrm{nV} *$ & $64 \mathrm{~g}$ & $82 \times 51 \times 14 \mathrm{~mm}$ \\
\hline COCHLEEG & 250 to $4000 \mathrm{~Hz}$ & & $139 \mathrm{nV}$ & $47 \mathrm{~g}$ & $66 \times 33 \times 23 \mathrm{~mm}$ \\
\hline MASTER & $1000 \mathrm{~Hz}$ & 12 bits & N/A** & Couple $\mathrm{kg}$ & Table-top setup \\
\hline
\end{tabular}

* Data apparently taken from TI's datasheet, by Smarting manual.

** Master's input noise is tricky to measure as this system needs several equipment to be fully functional.

different electrode count and placement, they interpolated the channel recordings over a mesh-head model. This is important because differences between systems could be attributed to electrode positions. For this study however, electrode count and placement remained the same over all systems, only the EEG amplifiers differed. Melnik et al.'s approach also took into consideration the different sources of variability and they found that variance across subjects was generally higher than variance across systems. Although this comparison is interesting when evaluating the importance of the difference in performance between systems, it was beyond the scope of this article. Another important comparison study between EEG systems was presented in De Vos et al.'s [6]. Their ERP study for a P300 speller application proposed as basis for comparison: EEG waveform, performance in spelling and information transfer rates (ITRs). These are in fact very relevant to consider when comparing systems for ERP studies. The ASSR paradigm however is a passive paradigm where the expected result is a frequency peak in the EEG spectrum.

The second study consisted of recording ERPs elicited with an active auditory oddball paradigm. This study was chosen to demonstrate CochlEEG's capability to record waveforms but was not a comparison between systems.

These studies were reviewed and approved by the Comité d'éthique pour la recherche, the Internal review Board at École de technologie supérieure (ÉTS) in Montreal, Canada. Informed consent was obtained from all participants before they were enrolled in the study.

\section{A. Participants}

Ten individuals (eight males, two females) aged between 22 and 34 and having hearing thresholds below $25 \mathrm{~dB}$ HL (pure tone audiometry between 125 to $8000 \mathrm{~Hz}$ ) participated in the ASSR study. Ten individuals (seven males, three females) with ages ranging from 21 to 34 and also with hearing thresholds below $25 \mathrm{~dB}$ HL participated in the ERP study. All participants were free of past or present neurological conditions.

\section{B. Hardware used}

\section{1) Hardware 1: MASTER system}

The Rotman MASTER (multiple auditory steady-state evoked response) research system is a data acquisition system designed by John \& Picton [7] to assess human hearing by recording ASSRs. This LabVIEW-based 
environment is capable of simultaneously generating multiple amplitude-modulated and/or frequency-modulated auditory stimuli, acquiring corresponding electrophysiological responses, displaying results in the frequency-domain, and determines whether the responses are significantly larger than background physiological activity. The MASTER system data acquisition setup for ASSR recordings is schematically illustrated in Figure 1.

ASSRs were filtered using a band-pass filter of $0.3-300 \mathrm{~Hz}$ (12 dB/octave) and amplified $50,000 \mathrm{X}(10,000 \mathrm{X}$ in GRASS Technologies LP511 AC Amplifier and 5X in National Instruments USB-6259 BNC). All data were collected using an Analog-to-digital (A/D) conversion rate of 1000 samples per second. In each recording, individual data epochs of 1024 points each were collected and connected together into sweeps (16 epochs per sweep of 16.384 seconds each). A typical ASSR recording for this study required a total of 6 sweeps over 98.304 seconds. To minimize artifacts, epochs containing signals exceeding $\pm 80 \mu \mathrm{V}$ were rejected.

ASSR sweeps of data were averaged in the time domain and then analyzed on-line in the frequency domain using the Fast Fourier Transform (FFT).

Amplitudes are expressed in nanovolts (nV). An F-ratio statistic is used by the MASTER system to evaluate the probability that the amplitude of the ASSR is significantly different from the average amplitude of the background noise in the adjacent frequencies (within \pm 60 bins of the modulation frequency). A response is considered to be present if $p<0.05$.

\section{2) Hardware 2: SMARTING}

SMARTING shown in Figure 2 is a 24-channel mobile EEG amplifier developed by mBrainTrain (Belgrade, Serbia). The system features two sampling frequencies $(250 \mathrm{~Hz}$ or $500 \mathrm{~Hz})$, a resolution of 24 bits and a bandwidth from DC to $250 \mathrm{~Hz}$. The weight and size of the version used are respectively 64 grams and $82 \times 51 \times 14 \mathrm{~mm}$. The amplifier unit includes a $3 \mathrm{D}$ gyroscope and a power supply for several hours of use. It transmits data wirelessly with Bluetooth (v2.1) protocol to a paired laptop, which was positioned in the audiometric booth at a distance of about two meters from the participant, well within the device's range of 10 meters. ASSRs were recorded during 98.304 seconds, which corresponds to 6 sweeps of 16.384 seconds each, with two available sampling rates $(250$ and $500 \mathrm{~Hz})$, by using the software provided by the manufacturer (Smarting Streamer version 2.2.2.0). Event markers were generated at the beginning and at the end of each stimulation using Lab Streaming Layer (LSL), a data acquisition and control framework developed at the Swartz Center for Computational Neuroscience (available: https://github.com/sccn/labstreaminglayer) [8] for recording stimulations and data streams with precise synchronizing performances.

Data and event markers were written into a common file format (.xdf).

Data were analyzed offline using MATLAB and EEGLAB [9]. ASSR data were filtered using a FIR band-pass filter

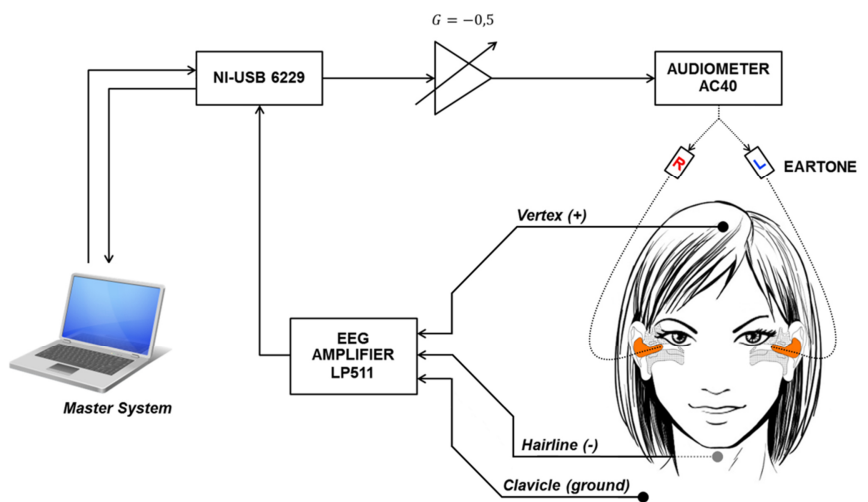

Fig. 1. Overview of a typical MASTER system setup. All components are monitored by a single PC. The stimulation signals from the analog output of the NI-USB 6229 board are attenuated by an operational amplifier with a gain of -0.5 , so that they may be delivered to the line input of the audiometer, which enables the operator to adjust the levels of stimuli delivered by the insert earphones. In parallel, ASSRs are scalp-recorded by the electrodes (placed between vertex (+) and hairline (-), with clavicle as a ground) and are then amplified by an EEG amplifier before reaching the analog input of the data acquisition board. Data are processed online through the MASTER system software.

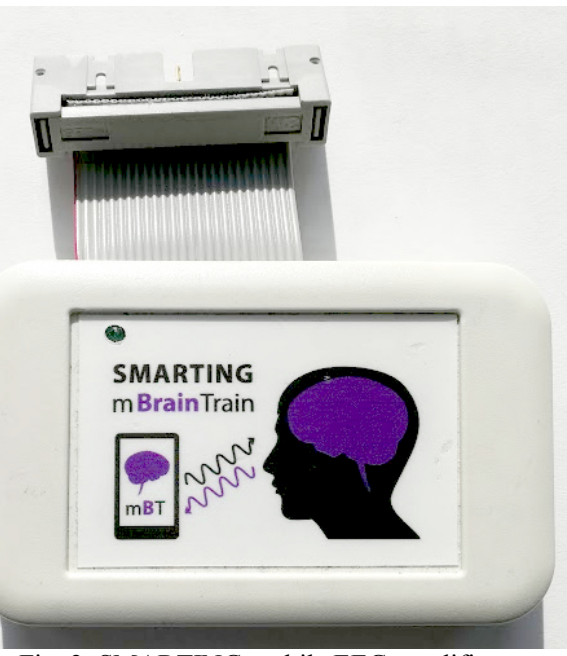

Fig. 2. SMARTING mobile EEG amplifier.

with an upper cut-off frequency of $0.1 \mathrm{~Hz}$ and lower cutoff frequency of half of the sampling rate. Sweeps of data were then extracted from the data stream using LSL markers and were averaged in the time domain before being translated in the frequency domain using FFT. The procedures of calculating amplitudes and background noise were similar to those used for the MASTER system.

\section{3) Hardware 3: CochlEEG}

CochlEEG, shown in Figure 3, is a custom 8-channel portable EEG research platform using a low-noise simultaneous 24-bit sampling analog-to-digital converter (ADS1299) developed by Texas Instruments. CochlEEG benefits from the use of this integrated chip by having user-configurable variable gain amplifiers, and variable sampling rates from $250 \mathrm{~Hz}$ to $4000 \mathrm{~Hz}$. CochlEEG's measured input-referred noise is $139 \mathrm{nV}_{\mathrm{RMS}}$ (using a linear gain of 24 and $250 \mathrm{~Hz}$ ). It has a bipolar $2.5 \mathrm{~V}$ supply output for active electrodes support and a mini-USB port with $5 \mathrm{kV}$ isolation for data transmission. The system is 47 


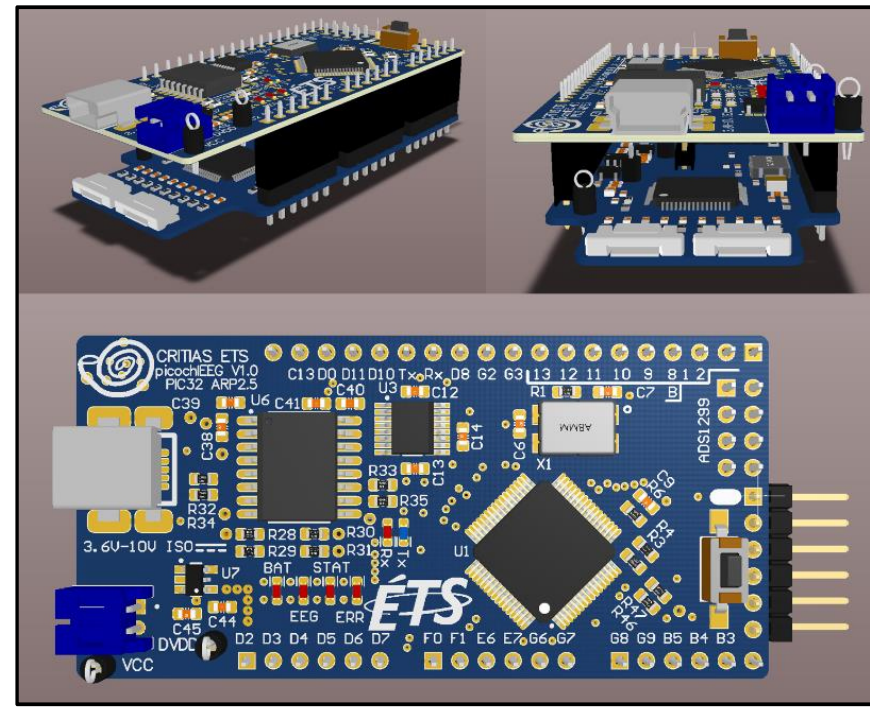

Fig. 3. 3D rendering of CochlEEG portable EEG amplifier (from [10])

grams in weight and measures $66 \times 33 \times 23 \mathrm{~mm}$, including the battery. CochlEEG can acquire EEG data for multiple hours without interruption. A block diagram of the hardware architecture is illustrated in Figure 4.

The current firmware version supports up to $4000 \mathrm{~Hz}$ sampling rate for 8 channels, it could support up to 16000 $\mathrm{Hz}$ for 8 channels pending minor hardware redesign.

CochlEEG offers highly configurable options using a custom LSL driver interface as shown in Figure 5. Each channel is independently configurable and global system settings such as EEG reference selection, sampling rate and timestamp source can be configured. The driver acts as an LSL stream publisher. Any LSL subscriber application is then free to listen to the published streams. CochlEEG timestamps every data conversion with a microsecond precision using an internal timer on a $40 \mathrm{MHz}$ bus clock to alleviate any possible data transmission timing issue. Internal timestamping of the EEG data reduces potential contamination of the signal by jitter or delay issues.

CochlEEG-recorded ASSRs were collected with sampling rates of 250, 500, 1000 and $2000 \mathrm{~Hz}$. The stimulation and recording were synchronized by using LSL. Data and event markers were written into a common file format (.xdf). Markers were generated in the beginning and at the end of each stimulation using LSL. Data processing was carried out offline using the same MATLAB script as for the SMARTING-recorded ASSR. Recording duration for task 3 was rigorously the same as that of tasks 1 and 2 (98.307 seconds which corresponds to 6 sweeps of 16.384 seconds each).

\section{Study 1: Auditory Steady-State Responses}

ASSR techniques have been largely developed to overcome the various difficulties in the assessment of hearing thresholds of very young children, older patients or those with cognitive deficits or behavioral disorders $[11,12]$. ASSRs are electrophysiological responses, recorded from the human scalp, and often evoked by one or more carrier frequencies $f_{c}$ that are amplitude-modulated at a specific frequency $f_{m}$. In practice, when a subject is

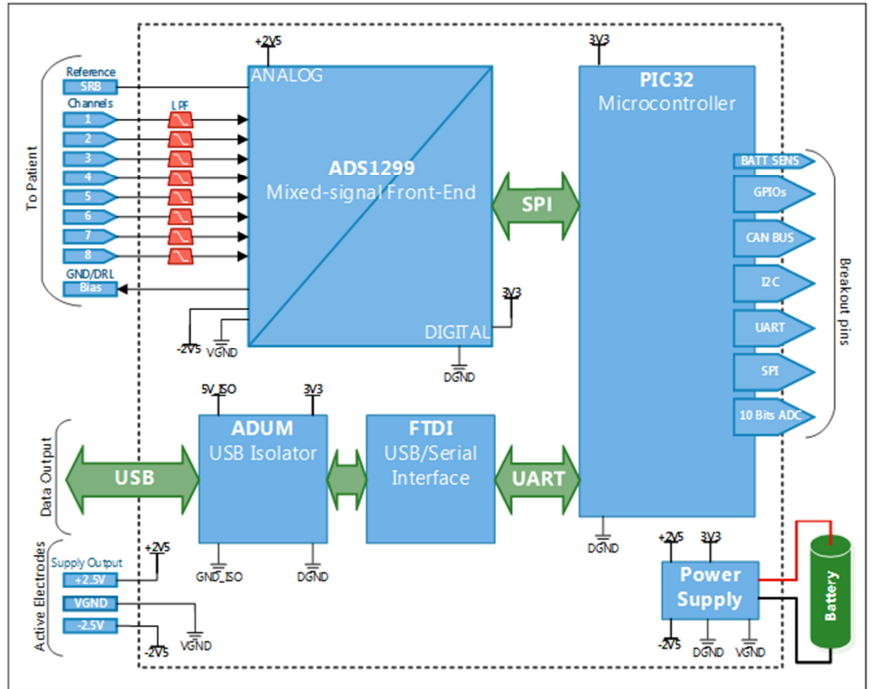

Fig. 4. CochlEEG block diagram showing the proper integration of the ADS1299 specialized chip (from [10])

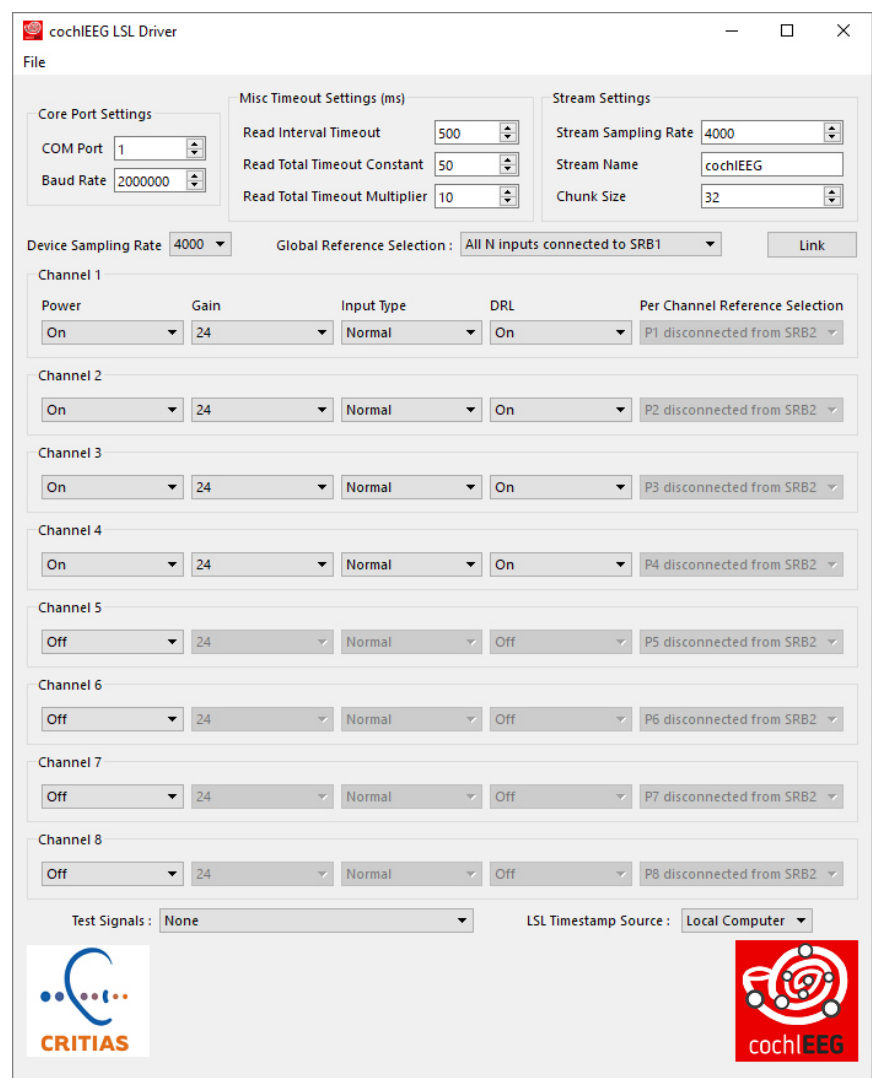

Fig. 5. Graphical User Interface (GUI) of the CochlEEG LSL driver showing the various channel configurations and streaming options.

exposed to such a stimulus, a peak in the frequency of their EEG will manifest at $\mathrm{f}_{\mathrm{m}}$, and may also appear at its harmonics [16].

Historically, ASSRs were first recorded at modulation frequencies near $40 \mathrm{~Hz}$ [13]. Since $40 \mathrm{~Hz}$-ASSRs are sensitive to arousal effects, a large number of studies using ASSRs for threshold estimation have concentrated on modulation frequencies in the range of $70-110 \mathrm{~Hz}$. In the case of alert/awake adults, modulation frequency at $40 \mathrm{~Hz}$ provides higher ASSR amplitudes and better signal-to- 
noise ratios, which may enable a faster and more efficient ASSR detection $[14,15]$.

ASSRs have been the subject of many studies so far, however, the capability of CochlEEG to record highquality ASSR signals is investigated in this research and the obtained results are compared with similar mobile and conventional research EEG recording systems.

\section{1) Stimuli}

ASSR stimuli used in this experiment consist of sinusoidal tones with the carrier frequencies of 500, 1000, 2000 and $4000 \mathrm{~Hz}$ that are all $100 \%$ amplitude modulated at $41 \mathrm{~Hz}$. Each amplitude-modulated stimulus is created by multiplying two sine waves. The sine wave with the higher frequency is the carrier frequency $f_{c}$ and the sine wave with the lower frequency forms represents the modulating envelope $f_{m}$. The full mathematical formula used to generate the stimuli $y(t)$ is given by:

$$
y(t)=\frac{\left[A^{*} \sin \left(2 \pi f_{c} t+\theta_{c}\right)\right]\left[\left(M * \sin \left(2 \pi f_{m} t+\theta_{m}\right)+1\right)\right]}{M+1}
$$

Where $A$ and $M$ are respectively the amplitude at the carrier frequency and the amount of modulation (from 0 to 1 ); $\theta_{c}$ and $\theta_{m}$ are the corresponding phase angles. The final stimuli were obtained by summing the individual amplitude-modulated stimuli. Individual stimuli characteristics are listed in table II.

Both carrier and modulation frequencies were softwareadjusted automatically so that an integer number of cycles occurred within each recording section. Consequently, individual sections can be linked without acoustic artifacts and are interchangeable during artifact rejection [16]. For simplicity, the frequencies will henceforth be reported to the nearest integer value. For example, a modulation frequency of $41.016 \mathrm{~Hz}$ is reported as $41 \mathrm{~Hz}$.

Stimuli were generated by either the MASTER system (for task 1) or MATLAB R2015b (for tasks 2 \& 3) and then were amplified by an Interacoustic ${ }^{\mathrm{TM}}$ audiometer (model AC40) before being presented binaurally to the testsubjects using E-A-RTONE ${ }^{\mathrm{TM}}$ insert earphones (model 3A 410-310). Test signals were calibrated at $75 \mathrm{~dB}$ SPL with a Bruël \& $\mathrm{Kjaer}^{\mathrm{TM}}$ Head and Torso Simulator (model 4128).

\section{2) Recordings}

All ASSR data were collected from an electrode placed at the vertex $(\mathrm{Cz})$ using an electrode on the back of the neck (below the hair-line) as reference and an electrode on the clavicle as ground. Electrodes were not removed between recordings and all interelectrode impedances were below 5 $\mathrm{k} \Omega$ at $10 \mathrm{~Hz}$. Data were first recorded using the MASTER system and then SMARTING subsequently. Finally, data were collected using CochlEEG. During all these measurements, test-subjects remained seated on a comfortable ergonomic chair inside a double-walled audiometric booth (Figure 6).

\section{Study 2: Event-related potentials}

Event-related potentials (ERP) are electrophysiological responses that are time-locked to an event (such as the
TABLE II

INDIVIDUAL STIMULI CHARACTERISTICS USED TO CREATE THE FINAL STIMULI

\begin{tabular}{|c|c|c|c|c|c|c|}
\cline { 3 - 7 } \multicolumn{1}{c|}{} & \multicolumn{6}{c|}{ Stimuli parameters } \\
\cline { 3 - 7 } \multicolumn{1}{c|}{} & $A$ & $f_{c}$ & $\theta_{c}$ & $f_{m}$ & $M$ & $\theta_{m}$ \\
\hline$\# \mathbf{1}$ & & $500 \mathrm{~Hz}$ & & & & \\
\hline $\mathbf{\# 2}$ & & $1000 \mathrm{~Hz}$ & 0 & $41 \mathrm{~Hz}$ & 1 & 0 \\
\hline $\mathbf{\# 4}$ & & $2000 \mathrm{~Hz}$ & & & & \\
\hline
\end{tabular}

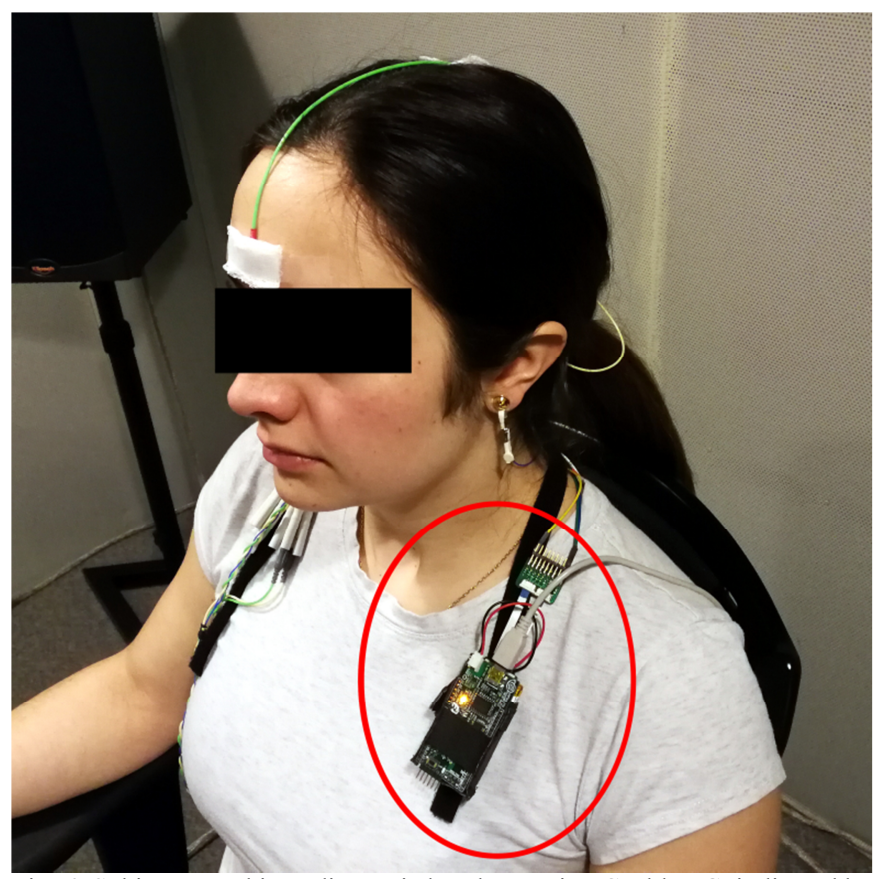

Fig. 6. Subject seated in audiometric booth, wearing CochlEEG, indicated by the red circle.

onset of a stimulus) and characterized by waveforms consisting of a series of positive and negative voltage deflections related to a set of components involved in neurocognitive processes [17].

Most of these voltage deflections are referred to by a letter indicating the polarity ( $\mathrm{N}$ for negative and $\mathrm{P}$ for positive) and by a number indicating the theoretical latency in milliseconds (or the ordinal position in the waveform). For instance, the N100 (or N1) wave is the first negative deflection peaking between 80 and $120 \mathrm{~ms}$ after the onset of an auditory stimulus and reflects the detection of a change in the acoustic environment [18].

The P300 (or P3) wave is the largest positive deflection, peaking between 250 and $500 \mathrm{~ms}$ after presentation of a task-relevant stimulus and reflects processes involved in decision-making. In a classic two-stimulus oddball task, participants are asked to respond to the target stimuli with a specific action while ignoring the standard stimuli. The P300 wave is typically observed around $300 \mathrm{~ms}$ after the onset of a target stimulus [19]. Auditory oddball paradigms use tonal stimuli presented in random order, with the target tone occurring less frequently than the standard tone.

ERP waveforms may also be referred to with acronyms as, for instance, the mismatch negativity (MMN) which is a brain response to violations of a rule, established by a sequence of sensory stimuli (typically in the auditory domain) [20]. The MMN reflects the brain's ability to 
perform automatic comparisons, detecting any change in a sequence of auditory stimuli, using traces developed by the previous stimulation. In a typical oddball task, the MMN is produced when deviant stimuli are presented in an otherwise homogeneous sequence of auditory stimuli; for instance, in a sequence of sounds " $\mathrm{a}$ a a a a $\mathrm{i}$ a a a $\mathrm{i}$ a a a...", the deviant stimulus "i" will elicit an MMN response. Contrary to the P300, the MMN is not taskdependent and can be triggered even when the participant is passive. Both the P300 and the MMN are studied using difference waves between target and non-target stimuli, and standard and deviant stimuli respectively. This isolates the studied phenomenon or wave from the rest of the EEG activity which should be the same for each recording.

Since ERP paradigms are widely used in brain-computer interfaces (BCI) [21], the capability of CochlEEG in recording reliable ERP waves is investigated in this research to illustrate the feasibility of using the proposed EEG acquisition platform for future BCI applications. Specifically the P300 was chosen since it reflects a conscious, voluntary cognitive process.

\section{1) Stimuli}

Auditory oddball stimuli consist of two pure-tone sounds $(1000 \mathrm{~Hz}$ and $2000 \mathrm{~Hz})$ of $69.8 \mathrm{~ms}$ duration $(9.9 \mathrm{~ms}$ rise/fall and $50 \mathrm{~ms}$ plateau). A total of 20 sequences of 10 tones were presented binaurally with a 2 seconds interstimulus interval at a participant-controlled comfortable loudness using E-A-RTONE ${ }^{\mathrm{TM}}$ insert earphones. The first six tones were always $1000 \mathrm{~Hz}$ standard tones, with a $2000 \mathrm{~Hz}$ target tone presented randomly at either the 7th, 8th, 9th or 10th position (standard tones were presented in the remaining non-target positions). A fixed inter-sequence interval of $4000 \mathrm{~ms}$ was used between recording sessions.

\section{2) Recordings}

Since the feasibility of using CochlEEG in ERP-based BCI applications is investigated in this study, a minimal number of electrodes was deliberately used to reproduce the limitations of acceptability of any device that is to be worn in social settings. Consequently, ERPs were recorded using the vertex $(\mathrm{Cz})$ as the active electrode, the left earlobes as the reference electrode and the forehead as the ground electrode; no electrooculogram electrode was used to correct eye-blink artefacts in the EEG. All interelectrode impedances were below $5 \mathrm{k} \Omega$ at $10 \mathrm{~Hz}$. No online filtering was applied (aside from the anti-aliasing filter implemented in the hardware) and no online rejection criteria were set. During all these measurements, testsubjects remained seated on a comfortable ergonomic chair inside a double-walled audiometric booth (see Fig. 6) and were instructed to silently count each occurrence of the target stimuli and to respond to the target stimuli by pressing a button when the target was detected.

For the analysis of the oddball data, continuous time series were band-pass filtered with a zero-phase finite impulse response filter between $0.1 \mathrm{~Hz}(-6 \mathrm{~dB}$ cut-off at $0.05 \mathrm{~Hz}$, filter order 33000$)$ and $20 \mathrm{~Hz}(-6 \mathrm{~dB}$ cutoff at $20.015 \mathrm{~Hz}$, order 33000) and afterwards epoched from - 200 to $700 \mathrm{~ms}$ and baseline corrected ( -200 to $0 \mathrm{~ms}$ ).

\section{RESULTS}

Figure 7 presents the average ASSR signal-to-noise ratios (SNRs) computed on ten test-subjects for each recording system. Examples of individual ASSR results recorded with the MASTER system, the SMARTING and CochlEEG are shown in Figure 8. The grand average ERP is illustrated in Figure 9.

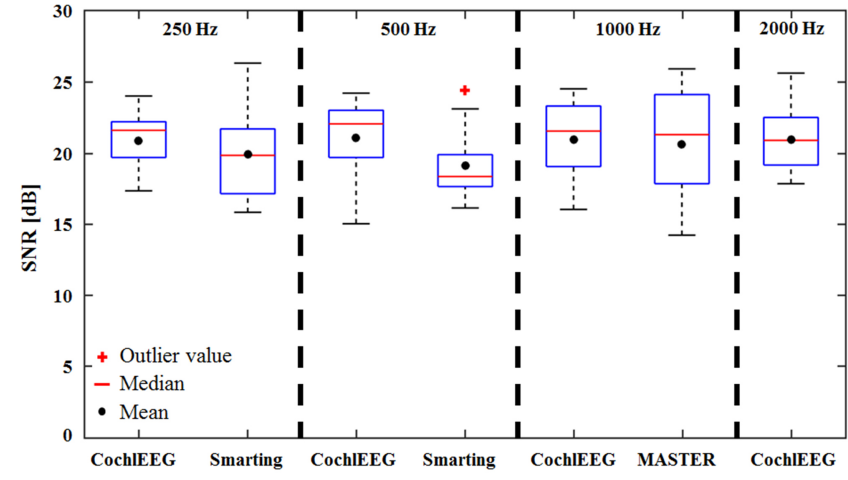

Fig. 7. Average ASSR signal-to-noise ratios, in $\mathrm{dB}$, computed on ten subjects.
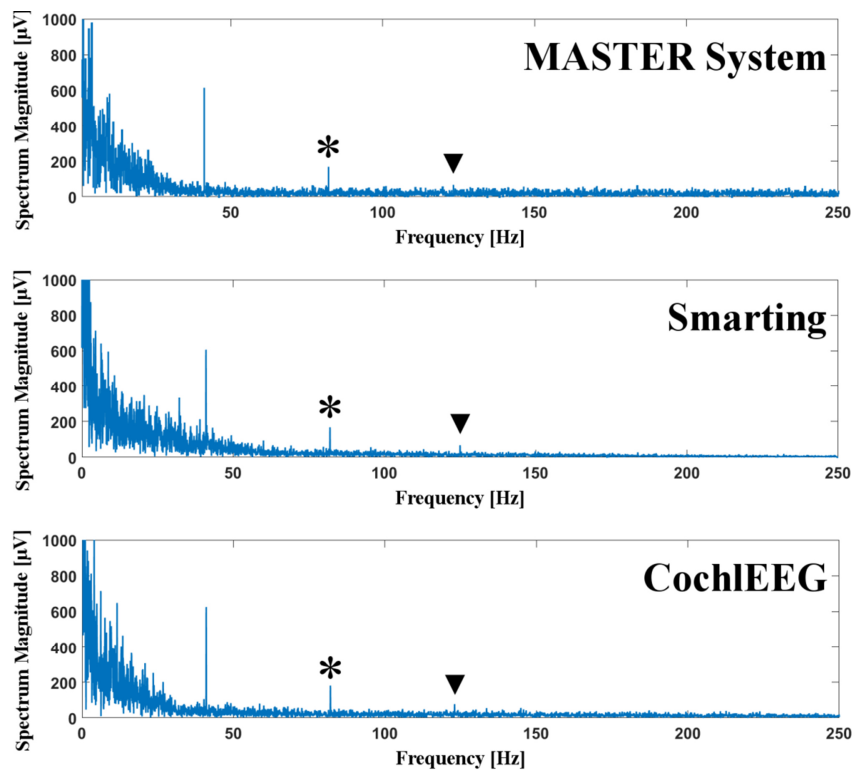

Fig. 8. Power spectrum of EEG signals recorded on subject \#1 with the MASTER system (top), the SMARTING with a $500 \mathrm{~Hz}$ sampling frequency (middle) and CochlEEG with a $1 \mathrm{kHz}$ sampling frequency (bottom). Peaks indicated with asterisks and solid triangles correspond to the second and third harmonics of the $41 \mathrm{~Hz}$ responses, which are particularly visible in this subject.

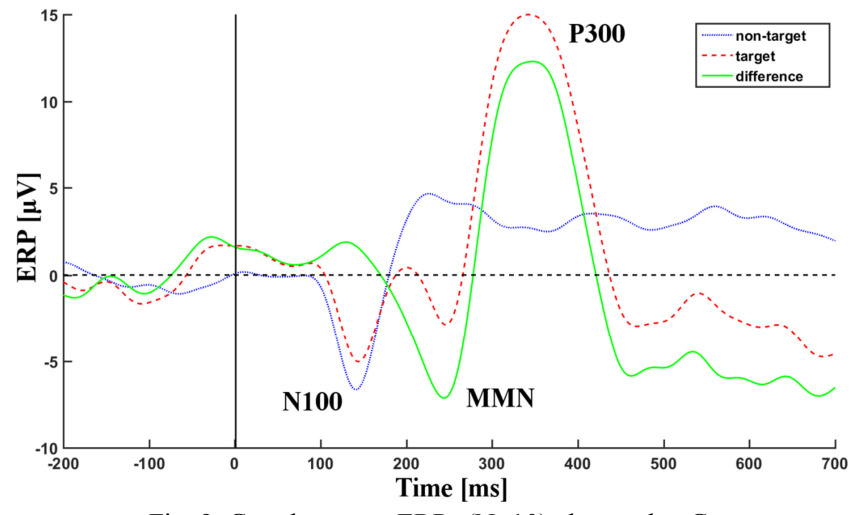

Fig. 9. Grand average ERPs $(\mathrm{N}=10)$ observed at $\mathrm{Cz}$. 
The low target detection error rates for the ERP study (mean $=0.1 \%$ ) demonstrates that subjects were able to easily distinguish the target stimuli from the standard stimuli during the auditory oddball experiment.

Results in Figure 7 indicate that SNRs obtained using CochlEEG seem comparable to those obtained with the SMARTING at $250 \mathrm{~Hz}$ and $500 \mathrm{~Hz}$. Also, SNRs obtained using CochlEEG seem comparable to those obtained with MASTER system at $1000 \mathrm{~Hz}$. Furthermore, SNRs obtained using CochlEEG at $2000 \mathrm{~Hz}$ seem comparable to those obtained with CochlEEG at $1000 \mathrm{~Hz}, 500 \mathrm{~Hz}$ and $250 \mathrm{~Hz}$ sampling rates.

These observations are confirmed by Wilcoxon tests, computed using R 3.1.0 with MASS 7.3-35 [22]. These tests fail to reject the null-hypothesis at a 5\% significance level at $250 \mathrm{~Hz}$ for SNRs obtained using CochlEEG and SMARTING $(p=0.241)$, at $500 \mathrm{~Hz}$ for SNRs obtained using CochlEEG and SMARTING $(p=0.054)$ and at $1 \mathrm{kHz}$ for SNRs obtained using CochlEEG and MASTER system $(p=0.919)$. The Wilcoxon tests give the same results when comparing SNRs obtained using CochlEEG at $2000 \mathrm{~Hz}$ and SNRs obtained using CochlEEG at $1000 \mathrm{~Hz} \quad(p=0.696)$, at $500 \mathrm{~Hz}$ $(p=0.838)$ and at $250 \mathrm{~Hz}(p=0.646)$. A two one-sided test procedure (TOST) [23] concludes that:

(i) At $250 \mathrm{~Hz}$, CochlEEG's SNRs and SMARTING's SNRs are equivalent at $\pm 4 \mathrm{~dB}(p<0.05)$.

(ii) At $500 \mathrm{~Hz}$, CochlEEG's SNRs and SMARTING's SNRs are equivalent at $\pm 5 \mathrm{~dB}(p<0.05)$.

(iii) At $1000 \mathrm{~Hz}$, CochlEEG's SNRs and MASTER system's SNRs are equivalent at $\pm 3 \mathrm{~dB}(p<0.05)$.

\section{DISCUSSION}

From the previous results, the fact that SNR results for CochlEEG and MASTER system are equivalent at $1000 \mathrm{~Hz}$ supports the idea that CochlEEG could be a suitable recording platform for ASSR. Indeed, MASTER System is a researchgrade, well-established ASSR recording platform being widely used and it is accepted as accurate. Additionally, the fact that SNR results for CochlEEG and SMARTING are equivalent at $250 \mathrm{~Hz}$ and $500 \mathrm{~Hz}$ supports the idea that these two devices, based on TI's ADS1299 chip, are comparable. Furthermore, ERP results demonstrate the feasibility of using CochlEEG in ERP-based applications. As shown in Figure 9, at Cz position, the N100 waveform is clearly identifiable and target ERPs evoked a large P300 and elicit a clear MMN response.

\section{CONCLUSION AND FUtURE WORK}

CochlEEG was presented in this paper as a light-weight EEG acquisition platform with the intention of enabling future innovative mobile EEG applications. The performance of CochlEEG was evaluated with a conventional ASSR recording platform (MASTER system), a mobile EEG amplifier (SMARTING) under two electrophysiological paradigms (ERPs and ASSRs). The results prove the capability of the developed EEG data acquisition platform to reliably record good quality EEG data. CochlEEG benefits from high sampling rates (theoretically up to $16 \mathrm{kHz}$ and currently tested up to $4 \mathrm{kHz}$, with an $8 \mathrm{kHz}$ sampling frequency in development). These features make CochlEEG suitable for data acquisition for various applications in BCI, assistive technologies, ubiquitous healthcare and wellness, telemedicine as well as nonmedical-oriented fields such as neurogaming. Furthermore, CochlEEG's higher sampling rate (up to $4000 \mathrm{~Hz}$ ) could lead to the development of several key applications in audiology such as objective assessment of hearing (click elicited ABR) or objective characterization of the speech processing in population with language-based learning disability (speech elicited ABR), to name a few. Nevertheless, several optimizations and improvements are required to gain access to this new realm of countless dailylife applications.

Future work should, in particular, address the following outstanding issues:

- CochlEEG is lightweight in pocket-sized packaging, easy to wear at the shoulder or clipped on a belt. However, data are currently transmitted to a computer through a USB cable. Consequently, a wireless data transmission stack should be added to CochlEEG to render it fully mobile.

- While CochlEEG has been validated with traditional wet electrodes, it has also to support the use of dry capacitive electrodes. Capacitive electrodes may have a lower sensitivity and specificity than existing wet electrodes, but they do not require the abrasion of the Stratum Corneum and the use of electrolytic gel to reduce the skin impedance, which makes them more acceptable to users in social settings [24]. Nevertheless, CochlEEG's ability to accurately and precisely record EEG signals using dry capacitive electrodes needs to be further investigated.

- Despite the recent development of a small, wireless and lightweight head-mounted EEG system [25], EEG caps are still uncomfortable to wear and not suitable for dailylife situations. Consequently, CochlEEG should be compatible and tested with the next generation of inconspicuous EEG sensors acceptable in social settings as the cEEGrid [26] or the EARtrodes [27], to name a few examples.

\section{ACKNOWLEDGMENT}

The authors wish to acknowledge the financial support received from the NSERC-EERS Industrial Research Chair in In-Ear Technologies (CRITIAS), as well as from MITACS Elevation program and the technical support received from EERS Global Technologies.

\section{REFERENCES}

[1] Patel S, Park H, Bonato P, Chan L, Rodgers M. A review of wearable sensors and systems with application in rehabilitation. Journal of NeuroEngineering and Rehabilitation. 9(12):21, 2012. 
[2] Feng S, Tang M, Quivira F, Dyson T, Cuckov F, Schirner G. EEGu2. Proceedings of the 27th International Symposium on Rapid System Prototyping Shortening the Path from Specification to Prototype RSP'16, 2016, pp. 19-25.

[3] Rahman KKM, Nasor M. Multipurpose low cost bio-daq system for real time biomedical applications. International Conference on Information and Communication Technology Research (ICTRC), 2015, pp. 286-289.

[4] Dufort G, Favaro F, Lecumberry F, Martin A, Oliver JP, Oreggioni J, Ramirez I, Seroussi G, Steinfeld L. Wearable EEG via lossless compression. 38th Annual International Conference of the IEEE Engineering in Medicine and Biology Society (EMBC), 2016, pp. 19951998.

[5] Melnik A, Legkov P, Izdebskil K, Kärcher SM, Hairston WD, Ferris DP, König P. Systems, Subjects, Sessions: To What Extent Do These Factors Influence EEG Data? Frontiers in Human Neuroscience. 30 March 2017. https://doi.org/10.3389/fnhum.2017.00150

[6] De Vos M, Kroesen M, Emkes R, Debener S. P300 speller BCI with a mobile EEG system: comparison to a traditional amplifier. Journal of Neural Engineering, 11(3):1-8, 2014.

[7] John MS, Dimitrijevic A, Picton TW. MASTER: a Windows program for recording multiple auditory steady state responses. Computer Methods and Programs in Biomedicine. 61, 125-150, 2000.

[8] Bigdely-Shamlo N, Kreutz-Delgado K, Robbins K, Miyakoshi M, Westerfield M, Bel-Bahar T, Kothe C, Hsi J, Makeig S. Hierarchical Event Descriptor (HED) tags for analysis of event-related EEG studies. IEEE Global Conference on Signal and Information Processing, 2013, pp. 1-4. https://doi.org/10.1109/GlobalSIP.2013.6736796

[9] Delorme A, Makeig S. EEGLAB: An open source toolbox for analysis of single-trial EEG dynamics including independent component analysis. Journal of Neuroscience Methods. 15(134):9-21, 2004. Doi: 10.1016/j.jneumeth.2003.10.009.

[10] Ducharme M. Développement d'une plateforme de recherche portable pour électroencéphalographie intra- et circum-auriculaire. Master's thesis (publication pending). École de technologie supérieure Université du Québec, 2018.

[11] Cone-Wesson B, Dowell RC, Tomlin D, Rance G, Ming WJ. The auditory steady-state response: Comparisons with the auditory brainstem response. J Am Acad Audiol. 13:173-83, 2002.

[12] Picton TW, John MS, Dimitrijevic A, Purcell D. Human auditory steady state responses. Int J Audiol. 42:177-219, 2003.

[13] Galambos R, Makeig A, Talmachoff PJ. A 40-Hz auditory potential recorded from the human scalp. Proceedings of the National Academy of Sciences of the United States of America. 78(4):2643-47, 1981.
[14] Dimitrijevic A, John MS, van Roon P, Picton TW. Human auditory steady-state responses to tones independently modulated in both frequency and amplitude. Ear Hear. 22:100-11, 2001.

[15] Van Maanen A, Stapells DR. Comparison of multiple auditory steady state responses $(80$ versus $40 \mathrm{~Hz}$ ) and slow cortical potentials for threshold estimation in hearing-impaired adults. Int J Audiol. 44:613-24, 2005.

[16] John MS, Lins OG, Boucher BL, Picton TW. Multiple auditory steadystate responses (MASTER): Stimulus and recording parameters. Audiology. 37:59-82, 1998

[17] Luck SJ, Kappenman ES. The Oxford Handbook of Event-Related Potential Components, eds. (2012). Oxford University Press.

[18] Näätänen R, Picton T. The N1 wave of the human electric and magnetic response to sound: a review and an analysis of the component structure. Psychophysiology. 24(4):375-425, 1987.

[19] Donchin E, Coles MG. Is the P300 component a manifestation of context updating? Behav. Brain Sci. 11:357-427, 1988.

[20] Näätänen R, Winkler I. The concept of auditory stimulus representation in cognitive neuroscience. Psychological Bulletin. 125:826-859, 1999.

[21] Graimann B, Allison B, Pfurtscheller G. Brain-computer interfaces. Heidelberg, 2010. Germany: Springer. Doi: 10.1007/978-3-642-020919.

[22] R Core Team (2014). R: A language and environment for statistical computing. R Foundation for Statistical Computing, Vienna, Austria. ISBN 3-900051-07-0, URL http://www.R-project.org/

[23] Schuirmann DJ. A comparison of the two one-sided tests procedure and the power approach for assessing the equivalence of average bioavailability. J Pharmacokinet Biopharm. 15:657-80, 1987.

[24] Lin CT, Liao LD, Liu YH, Wang IJ, Lin BS, Chang JY. Novel dry polymer foam electrodes for long-term EEG measurement. IEEE Trans. Biomed. Eng. 58(5):1200-1207, 2011.

[25] Kranczioch C, Zich C, Schierholz I, Sterr A. Mobile EEG and its potential to promote the theory and application of imagery-based motor rehabilitation, International Journal of Psychophysiology. 2013. http://dx.doi.org/10.1016/j.ijpsycho.2013.10.004

[26] Debener S, Emkes R, De Vos M, Bleichner M. Unobtrusive ambulatory EEG using a smartphone and flexible printed electrodes around the ear. Scientific Reports. 5:16743, 2015. Doi: 10.1038/srep16743

[27] Valentin O, Viallet G, Voix J. EARtrodes : towards a wireless in-ear custom fitted brain computeur interface. Canadian Acoustics Acoustique Canadienne. 45(3):142-3. 2017. 